\title{
FRACTALS BASED ON REGULAR CONVEX POLYTOPES
}

\author{
Andrzej Katunin \\ Department of Fundamentals of Machinery Design, Silesian University of Technology, Poland \\ andrzej.katunin@polsl.pl
}

\begin{abstract}
In the present paper the author presented new deterministic fractals based on regular convex polytopes, discussed their construction and presented special cases of some non-regular polytope-based fractals. Firstly, the fractals based on the regular convex polychora were investigated. Their construction was analyzed using different approaches and their Hausdorff dimension was determined. Then, the high-dimensional regular convex polytopes-based fractals were investigated. It was shown, that there is a possibility to determine and generalize the Hausdorff dimension of these fractals to $\mathbf{R}^{d}$. Moreover, two classes of fractals based on $d$-dimensional hyperpyramids and hyperbipyramids were discussed.
\end{abstract}

\section{Introduction}

Fractals based on one-, two- and three-dimensional polytopes are well-known and could be easily constructed. Moreover, there are strong connections between fractals in different dimensions, e.g. Cantor set - Sierpiński triangle - Sierpiński gasket or Cantor set - Sierpiński carpet - Menger sponge. It was shown, that there is a possibility of generalization of the construction of such fractals, which are based on regular polygons [1] and regular polyhedra [2]. It is easy to observe that the appropriate definition of the number of sides (for regular polygons) and the scale factor could give us well known fractals: Sierpiński triangle, Sierpiński carpet, Sierpiński pentagon etc. Following this we could extend these relationships to three-dimensional deterministic fractals and obtain Sieprinski gasket, Menger sponge and other polyhedra-based fractals.

The investigation of the fractals with higher dimension is related to the needs of topical scientific problems. The great impact of improvement of high-dimensional fractals is noticeable in quantum physics, especially in supersymmetric string theory and its modifications and simplifications. Research on this field was successfully provided by El Naschie. Many of his works (e.g. [3, 4]) are concentrated on describing the high energy physics problems using uniform convex polytopes. The four-dimensional fractals also could find an application in problems of the multidimensional signal processing. Some works in this area $[5,6]$ present the four-dimensional wavelet packets for application in the multidimensional signal processing. Finally, there is a group of problems related to the visualization of 
high-dimensional data including quaternionic and octonionic complex fractals (e.g. Mandelbrot and Julia set generalized to high-dimensional space). Quaternions also found an application in problems of signal encryption [7]. In available literature some of multidimensional fractals were already presented, e.g. Sierpiński triangle in higher dimensions [8], high-dimensional Hilbert cube [9] or the hypergasket [10]. The aim of this work is the generalization of the regular convex polytopesbased fractals and determination of the relationships of their Hausdorff dimension.

Let us define the fractal in $\mathbf{R}^{d}$, which is the attractor $A_{\infty}^{S}$ of the IFS with appropriate parameters, where $S$ in the superscript denotes the Schläfli symbol of a given polytope and infinity in the subscript denotes the number of iterations. The most traditional method of the construction of self-similar fractals is the method of successive approximations, which is based on the definition of IFS with appropriate contraction mappings. Let $V \subset \mathrm{R}^{d}$, where $V$ is the set of $z$ vectors, which contain the coordinates of the vertices $v_{i}$ of a given polytope $A_{0}^{S}$. Then, we define the elementary similarity transformation in the form of:

$$
w_{i}(v)=r v+(r-1) v_{i} \text { for } 0<r<1,1 \leq i \leq n,
$$

where $r$ is the scale factor. Describing the contraction of $A_{k}$ to $A_{k+1}$ (where $k \geq 0$ ) we use the Hutchinson operator:

$$
W\left(A_{k+1}^{S}\right)=\bigcup_{i=1}^{n} w_{i}\left(A_{k}^{S}\right)
$$

Definition 1. Let $A_{0}^{S}$ be a regular convex d-polytope in the Euclidean space $\mathbf{R}^{d}$. The set

$$
A_{\infty}^{S}=\bigcap_{i=0}^{\infty} w^{i}\left(A_{0}^{S}\right)
$$

where $w_{i}\left(A_{k}^{S}\right) \cap w_{j}\left(A_{k}^{S}\right)=\varnothing, \quad i \neq j$ is called the fractal in $\mathbf{R}^{d}$ of $A_{0}^{S}$ with the unique scale factor $r$.

\section{Regular convex polychora-based fractals}

There are six regular polytopes in $\mathbf{R}^{4}$, which are presented in Figure 1 in a hyperspherical projection (using Jenn3d software).

Let us consider the first polychoron - the pentachoron (also called simplex or 5-cell). The fractal, which based on a pentachoron, whose attractor $A_{\infty}^{\{3,3,3\}}$ we will call the hypergasket due to the nomenclature used e.g. in $[10,11]$. Let $A_{0}^{\{3,3,3\}}$ be a regular unitary simplex. After the first iteration we obtain 6 simplexes on the 
base and 1 at the apex with edge-length $1 / 2$. Thus, the scale factor is $r=2$. After $k$ iterations we obtain $7^{k}$ simplexes with the sides of length $2^{-k}$. Considering this, the Hausdorff dimension of a hypergasket could be determined using the well-known formula:

$$
D_{H}=\frac{\log (N)}{\log (r)}
$$

where $N$ is the number of sub-elements of the fractal on the $k$-th level of approximation. The Hausdorff dimension of the hypergasket equals $\log (7) / \log (2) \approx$ $\approx 2.80735$.

a)

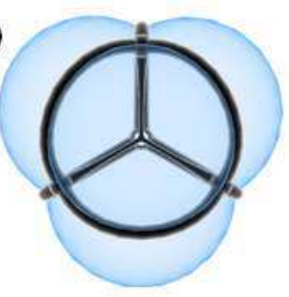

d)

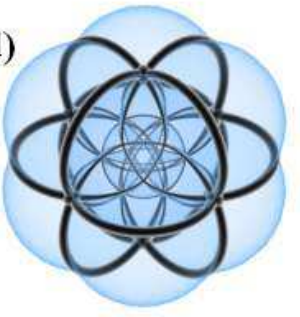

b)

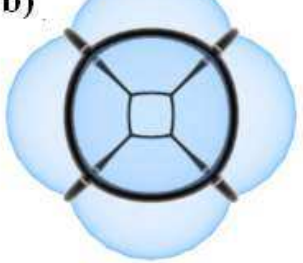

e)

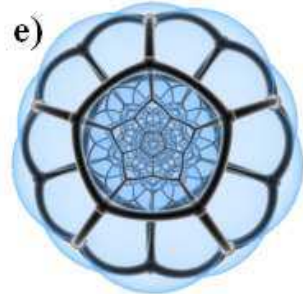

c)
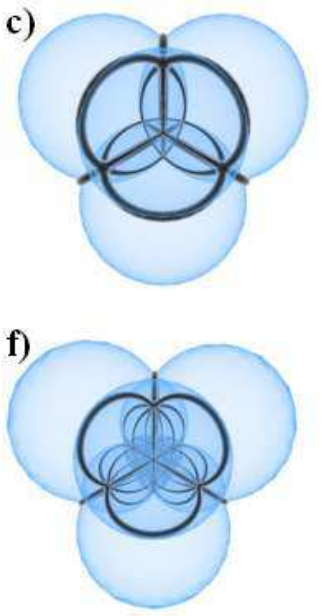

Fig. 1. Regular polychora: a) pentachoron, b) octachoron, c) hexadecachoron, d) icositetrachoron, e) hecatonicosachoron, f) hexacosichoron

Next we define the hypersonge, a four-dimensional fractal based on the octachoron (hypercube or 8-cell). The analogous fractal to the hypersponge is the Sierpiński carpet in two dimensions and the Menger sponge in three dimensions. Considering this, we could conclude that the scale factor $r=3$, the same as for the hypersponge's lower-dimensional analogs. For the determination of the number of 1/3-times scaled contractions we use a different approach - the addressing system presented in [12] for the Cantor set and Sierpiński triangle. Let us consider the hypercube with edge-length equal to 3 . Let us use the set $\{1,2,3\}$ for creation of addresses of sub-hypercubes, which determine the position of the element in $\mathbf{R}^{4}$. It is evident, that we will obtain 81 four-symbol addresses. Analyzing analogs of $A_{\infty}^{\{4,3,3\}}$ using the above-presented addressing system we could conclude, that for obtaining the fractal we exclude the elements, whose addresses contain at least two 
" 2 ": for the Sierpiński carpet it is 1 and for the Menger sponge it is 7 . It is easy to calculate that for the hypersponge we obtain 33 such addresses. Having the number of sub-hypercubes after the first iteration we could calculate its Hausdorff dimension: $\log (48) / \log (3) \approx 3.52372$.

The third 4-polytope from the group of convex polychora is the hexadecachoron (orthoplex or 16-cell). The fractal based on this polytope we will be called the hyperbigasket. It could be noticed that each facet of the hyperbigasket is the Sierpiński octahedron, thus the scale factor $r=2$. As it could be observed, there is some similarity between the hyperbigasket and the hypergasket, which reflected in the construction of the hyperbigasket. During the iteration process we obtain 6 hexadecachora in a mid-space and 2 hexadecachora at the upper and lower vertices, thus the Hausdorff dimension of the hyperbigasket is $\log (8) / \log (2)=3$.

The icositetrachoron (hyperdiamond, octaplex or 24-cell) is the most unique polychoron, because it has no direct analog in any other dimension. The fractal constructed based on this polychoron we will call an octaplex fractal. The construction of this fractal is not so intuitively as in the previous cases. The 24 cells of the icositetrachoron are connected in the 24 vertices given by permutations of $( \pm 1$, $0,0,0)$ and $( \pm 0.5, \pm 0.5, \pm 0.5, \pm 0.5)$ with edge-length of 1 . For determining the scale factor for $A_{\infty}^{\{3,4,3\}}$ we use the parameter $\delta$, which is the ratio of the distance between the center of $A_{0}^{\{3,4,3\}}$ to whichever of its vertices (while the polytope is orthogonally projected onto a plane) and the edge-length of the polytope [13]. The scaling factor could be calculated from the next expression:

$$
r=1+\frac{{ }_{0} R}{l}=1+\delta
$$

Following Coxeter [13] $\delta=2$, thus the scaling factor for the octaplex fractal $A_{\infty}^{\{3,4,3\}}$ is $r=3$. Having the scaling factor we could determine the number of suboctaplexes after the first iteration. The icositetrachoron has 24 octahedral cells, which are connected in 24 vertices [13], whose coordinates are given by the abovepresented permutations. Considering that $r=3$ we obtain 72 vertices of $A_{1}^{\{3,4,3\}}$ which connect 72 sub-octaplexes with edge-length of $1 / 3$. Hence, the Hausdorff dimension of the octaplex fractal is $\log (72) / \log (3) \approx 3.89279$.

The hecatonicosachoron (dodecaplex or 120-cell) and the hexacosichoron (tetraplex or 600-cell) are also unique polychora, which have no analogs in higher dimensions. It is possible to construct two last fractals in 4-dimensional Euclidean space, which we will call the dodecaplex fractal and the tetraplex fractal, respectively. The hecatonicosachoron $A_{0}^{\{5,3,3\}}$ has 120 dodecahedral cells connected by 600 vertices. Their coordinates could be obtained by the multiplication of the centers of vertex figures (tetrahedra) by $4 \varphi^{-2}$, where $\varphi=(\sqrt{5}+1) / 2$ is the golden ratio. 
They include all permutations of $( \pm 2, \pm 2,0,0),( \pm \sqrt{5}, \pm 1, \pm 1, \pm 1),( \pm \varphi, \pm \varphi, \pm \varphi$, $\left.\pm \varphi^{-2}\right),\left( \pm \varphi^{2}, \pm \varphi^{-1}, \pm \varphi^{-1}, \pm \varphi^{-1}\right)$ and all even permutations of $\left( \pm \varphi^{2}, \pm 1, \pm \varphi^{-2}, 0\right),( \pm \sqrt{5}$, $\left.\pm \varphi, \pm \varphi^{-1}, 0\right),\left( \pm 2, \pm \varphi, \pm 1, \pm \varphi^{-1}\right)$. The edge-length of this polychoron is the distance between two neighboring vertices $\left(\varphi^{2}, \pm \varphi^{-2}, 1,0\right)$ and equals $2 \varphi^{-2}$. The parameter $\delta=\sqrt{8} \varphi^{2}$ [13]. By the analogy of similar fractals in lower dimensions [1,2] we put $\delta$-times reduced copies of $A_{0}^{\{5,3,3\}}$ in each vertex. Finally, the Hausdorff dimension of the dodecaplex fractal is $\log (600) / \log \left(1+\sqrt{8} \phi^{2}\right) \approx 3.00492$.

For the construction of the tetraplex fractal $A_{\infty}^{\{3,3,5\}}$ we use the same rule as before. The hexacosichoron with an edge-length of $2 \varphi^{-1}$ could be constructed from the next permutations of its 120 vertices: 96 even permutations of $\left( \pm \varphi, \pm 1, \pm \varphi^{-1}\right.$, $0), 8$ permutations of $( \pm 2,0,0,0)$ and 16 permutations of $( \pm 1, \pm 1, \pm 1, \pm 1)$. The parameter $\delta$ equals $2 \varphi$. Thus, the Hausdorff dimension of $A_{\infty}^{\{3,3,5\}}$ is $\log (120) / \log (1+2 \varphi) \approx 3.31627$.

\section{Fractals based on the regular convex polytopes in $\mathbf{R}^{d}$}

In the $d$-dimensional Euclidean space, where $d \geq 5$ there are only three kinds of the regular convex polytopes: $d$-simplex, $d$-hypercube and $d$-orthoplex. Based on these polytopes one could construct hyperfractals and generalize their Hausdorff dimension.

The $d$-simplex $A_{0}^{S}$ could be constructed similarly as its analogs in lower dimensions. Considering used nomenclature we will call such hyperfractals as $d$-hypergaskets. Note that during iteration there is always one $d$-sub-simplex at the apex of a $d$-simplex. $d$-sub-simplices on the base of a $d$-simplex grow twice with every higher dimension. The scaling factor is constant regardless of $d$ and equals 2 . Therefore, the Hausdorff dimension for $d$-hypergasket with any $d$ is:

$$
D_{H}=\frac{\log (3 d-5)}{\log 2} \text {. }
$$

Similarly, the other two above-mentioned hyperfractals could be generalized to $\mathrm{R}^{d}$. The d-hyperbigaskets are constructed in the same way as previously. The only difference in the Hausdorff dimension is two "stable" vertices of this polytope. The Hausdorff dimension formula for this class of hyperfractals is a slight modification of (6):

$$
D_{H}=\frac{\log (3 d-4)}{\log 2}
$$


The Hausdorff dimension of the d-hypersponge will be determined using the above-presented addressing approach. The scale factor for this hyperfractal is constant in every dimension and equals 3 .

Theorem 1. The number of contractions $N$ of the $d$-hypersponge in iteration $k$ is

$$
N_{k}^{d}=\left(3^{d}-\sum_{i=1}^{p} \frac{d !}{\prod_{j=1}^{q} e_{i j} !}\right)^{k}, p=f\left(C_{3}^{d}\right),
$$

where $f\left(C_{3}^{d}\right)$ denotes all combinations of addresses with at least two " 2 ".

Proof. Using the addressing approach for the $d$-hypercube it is possible to define $3^{d}$ addresses with elements $e_{j}$, where $j=1 \ldots d$, from the set $\{1,2,3\}$. From the obtained addresses one needs to exclude elements, which do not consist of the $d$-hypersponge. The addresses of these elements consist at least two "2" in any combination. Thus, the number of the elements to be excluded could be presented as a sum of permutations with repetitions of the $d$-element addresses. The difference between $3^{d}$ and obtained number raised to the $k$-th power gives the number of elements of $d$-hypersponge in $k$-th approximation level, which ends the proof.

Considering the Theorem 1 it is possible to obtain an expression for determination of Hausdorff dimension in a generalized form:

$$
D_{H}^{d}=\frac{\log \left(N^{d}\right)}{\log (3)},
$$

where $N^{d}$ is given by (8).

Now, let us analyze the character of changing the Hausdorff dimension of hyperfractals based on the regular convex polytopes depending on their dimension. Figure 2 presents above-mentioned dependence for $d$-hypergasket, $d$-hyperbigasket and $d$-hypersponge. As it could be noticed, the Hausdoff dimension of $d$-hypergasket and $d$-hyperbigasket becomes equal when $d \rightarrow \infty$ :

$$
\lim _{d \rightarrow \infty} \frac{\log (3 d-5)}{\log 2}=\lim _{d \rightarrow \infty} \frac{\log (3 d-4)}{\log 2}=\infty .
$$

Let us consider some special cases of $d$-simplex and $d$-orthoplex. If we consider them in three dimensions we obtain the tetrahedron and triangular bipyramid, whose base is a regular triangle. Bases of these polyhedra could be extended to regular convex $n$-gons and we obtain a class of $n$-gonal-based polyhedra. Determining parameters of the contraction it is possible to construct a new class of $n$-gonal-based fractals. Similarly, we could generalize the above-presented class to 
the higher dimensions. Two groups of hyperfractals based on $n$ - $d$-hyperpiramid and $n$ - $d$-hyperbipyramid we define as follows.

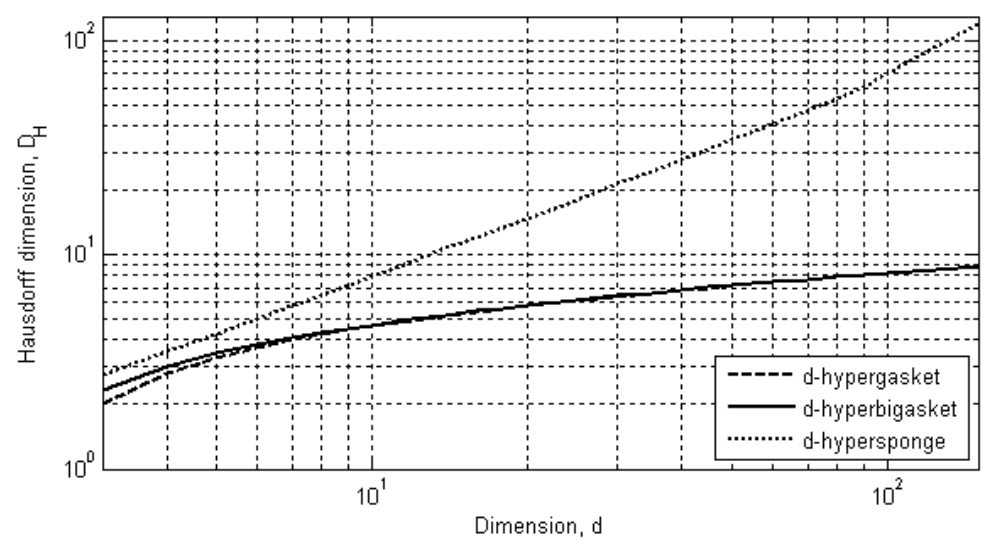

Fig. 2. Hausdorff dimension of the hyperfractals versus space dimension

Definition 2. Let us consider the $n$ - $d$-hyperpyramid $A_{0}^{P}$ and the $n$ - $d$ hyperbipyramid $A_{0}^{b P}$ in the Euclidean space $\mathbf{R}^{d}$, where $d \geq 3$. The set (3) is called the $n$ - $d$-hyperpyramid fractal constructed from $A_{0}^{P}$ and the $n$ - $d$-hyperpyramid fractal constructed from $A_{0}^{b P}$ with the unique scale factor $r_{n}$, which could be determined using following expression (cf. [1]):

$$
r_{n}=2\left(1-\frac{\tan \left(\frac{\pi}{n}\left\lfloor\frac{n-1}{4}\right\rfloor\right)}{\tan \left(\frac{\pi}{n}+\frac{\pi}{n}\left\lfloor\frac{n-1}{4}\right\rfloor\right)}\right)^{-1} .
$$

Based on the Definition 2 we could construct general formulas for determining the Hausdorff dimension for whichever hyperfractal from the above-presented groups. Analyzing Hausdorff dimension formulas for $d$-hypergasket and $d$-hyperbigasket one could conclude that the Hausdorff dimension for their $n$-gonal analogs is dependent on $n$. Thus, we could write such relations as follows:

$$
\begin{aligned}
& D_{H}^{P}=\frac{\log (n(d-2)+1)}{\log \left(r_{n}\right)}, \\
& D_{H}^{b P}=\frac{\log (n(d-2)+2)}{\log \left(r_{n}\right)} .
\end{aligned}
$$


Let us investigate the character of changing of Hausdorff dimension for two above-presented infinite groups of hyperfractals.

Theorem 2. The Hausdorff dimension of the $n$ - $d$-hyperpyramid fractal and the $n$ - $d$ hyperbipyramid fractal in $\mathbf{R}^{d}$ for any $d$ tends to unity, when $n$ tends to infinity.

Proof. When $n \rightarrow \infty$ the $n$-gonal bases of the given hyperfractal contractions tend to circles, thus the Haussdorf dimension of given hyperfractal tends to the Hausdoff dimension of the circle:

$$
\lim _{n \rightarrow \infty} \frac{\log (n(d-2)+1)}{\log \left(r_{n}\right)}=\lim _{n \rightarrow \infty} \frac{\log (n(d-2)+2)}{\log \left(r_{n}\right)}=1 .
$$

When we analyze the case when $n \rightarrow \infty$ and $d \rightarrow \infty$ we obtain the undefined value, because the Hausdorff dimension increases with the increase of $d$ and decreases with increase of $n$. The surface plot in Figure 3 presents the dependence of the Hausdorff dimension on $n$ and $d$.

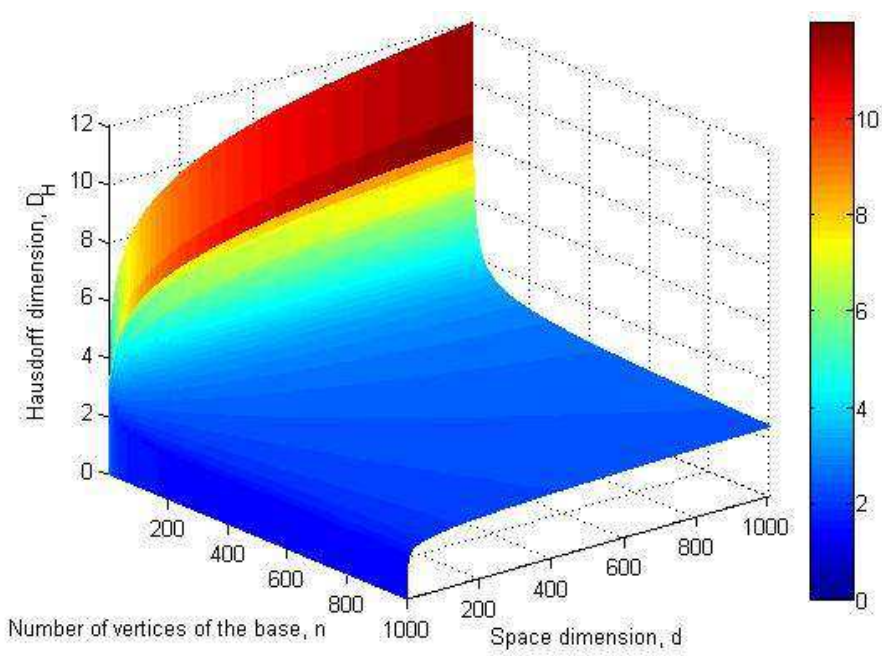

Fig. 3. Dependence of the Hausdorff dimension of the $n$ - $d$-hyperpyramid fractal and space dimension and number of vertices of the base of the contraction

\section{Conclusions}

In the paper the group of hyperfractals based on regular convex polytopes was presented. One determined the Hausdorff dimension of the fractals based on six regular convex polychora and their values varies as $3<D_{H}^{4}<4$. For the regular polytopes in higher Euclidean space dimensions, i.e. $d \geq 5$, the Hausdorff dimension expressions were generalized for each hyperfractal based on the $d$-polytope ( $d$-simplex, $d$-hypercube and $d$-orthoplex). Moreover, some special cases of hyper- 
fractals based on the $n$ - $d$-hyperpyramids and $n$ - $d$-hyperbipyramids were investigated. The Hausdorff dimension expressions for these groups were generalized for any $n \geq 3$ and any $d \geq 3$.

\section{References}

[1] Kahng B., Davis J., Maximal dimensions of uniform Sierpinski fractals, Fractals 2010, 18, 451$-460$.

[2] Kunnen A., Schlicker S., Regular Sieprinski polyhedra, Pi Mu Epsilon J. 1998, 10, 607-619.

[3] El Naschie M.S., Fuzzy Dodecahedron topology and E-infinity spacetime as a model for quantum physics, Chaos Soliton. Fract. 2006, 30, 1025-1033.

[4] El Naschie M.S., Average exceptional Lie and Coxeter group hierarchies with special reference to the standard model of high energy particle physics, Chaos Soliton. Fract. 2008, 37, 662-668.

[5] Tian X.X., Huo A.L., Construction and characteristics of a kind of four-dimensional vector wavelet packets with four-scale dilatation, Appl. Mech. Mater. 2010, 20-23, 1053-1059.

[6] Yu Y.M., Zhu Y.Q., Properties and constructing of a kind of four-dimensional vector wavelet packets according to a dilatation matrix, Key Eng. Mat. 2010, 439-440, 920-925.

[7] Nagase T., Komata M., Araki T., Secure signals transmission based on quaternion encryption scheme, Proc. of the $8^{\text {th }}$ International Conference on Advanced Information Networking and Applications (AINA'04), 2004.

[8] Brisson G.F., Reiter C.A., Sierpinski fractals from words in high dimensions, Chaos Soliton. Fract. 1995, 5, 2191-2200.

[9] He J.H., Hilbert cube model for fractal spacetime, Chaos Soliton. Fract. 2009, 42, 2754-2759.

[10] Altaisky M.V., Bednyakov V.A., Kovalenko S.G., On fractal structure of quantum gravity and relic radiation anisotropy, Int. J. Theor. Phys. 1996, 35, 253-262.

[11] Eyink G., Quantum field-theory models on fractal spacetime, Commun. Math. Phys. 1989, 125, 613-636.

[12] Peitgen H.-O., Jürgens H., Saupe D., Chaos and Fractals. New Frontiers of Science, SpringerVerlag, New York, Heidelberg 1991.

[13] Coxeter H.S.M., Regular Polytopes, Courier Dover Publications, 1973. 
